Special issue in honor of Prof. H.K. Lichtenthaler

\title{
The love-hate relationship between chlorophyll $a$ and water in PSII affects fluorescence products
}

\author{
C.R. GUADAGNO ${ }^{*+}$ (iD) , D.P. BEVERLY ${ }^{*, * *}$ (D) , and B.E. EWERS ${ }^{* * * *}$ (iD) \\ University of Wyoming, Department of Botany, Laramie, Wyoming, U.S.A.* \\ University of Wyoming, Water Resources/Environmental Science and Engineering, Laramie, Wyoming, U.S.A.* \\ University of Wyoming, Program in Ecology, Laramie, Wyoming, U.S.A. ${ }^{* * *}$
}

\begin{abstract}
Chlorophyll $a(\mathrm{Chl} a)$ has an asymmetrical molecular organization, which dictates its orientation and the location of the pigment in the mature photosynthetic apparatus. Although Chl $a$ fluorescence (ChlF) is widely accepted as a proxy for plant photosynthetic performance under countless stress conditions and across species, a mechanistic understanding of this causality is missing. Since water plays a much greater role than solvent for the photosynthetic machinery, elucidating its influence on $\mathrm{Chl} a$ may explain the reliable reflection of plant stress response in the ChlF signal. We examine the effect of hydration from well-watered to lethal drought on ChlF imagery results across morphologically diverse species to begin testing the impact of molecular scale hydration of Chl $a$ on ChlF. Our results support a conceptual model where water is an integral part of the photosystems' structure and directly influences Chl $a$ behavior leading to changes in the energy partitioning and ultimately in ChlF.
\end{abstract}

Keywords: chlorophyll a fluorescence; drought; leaf water content; molecular water; mortality; photosystem II.

\section{Introduction}

Chlorophylls are the most abundant pigments on Earth with Chl a making up approximately $75 \%$ of these complex chromophores (Guidi et al. 2017), so revealing their molecular stress response will improve the predictive understanding of photosynthesis. In photooxygenic organisms, Chl $a$ is essential to light absorption and to release chemical energy through the thylakoid membranes via photosynthesis (Raven et al. 2004). In higher plants, Chl a molecules are mainly organized with $\mathrm{Chl} b$ and other accessory pigments (e.g., carotenoids) to form the antenna complexes, the light-harvesting complex of photosystem I and II, LHCI and LHCII, respectively (Mirkovic et al. 2017). These are the sites where the photons of light arrive, causing electrons $\left(\mathrm{e}^{-}\right)$in the pigments to move from a ground state to a higher energetic level. The presence of diverse pigments, highly packed in the LHCs ensures that

\section{Highlights}

- ChlF imagery is affected by leaf hydration across extreme morphophysiological species

- Computed imaging ChlF highly correlates with relative water content from mild to lethal drought

- A model using molecular waters as an integral part of PSII structure can improve imaging $\mathrm{ChlF}$ analyses
Received 21 December 2020

Accepted 31 March 2021

Published online 25 May 2021

${ }^{+}$Corresponding author

e-mail: cguadagn@uwyo.edu

Acknowledgments: We thank Rachel Shrode, Ethan Darling, Keegan Ferris, and Hunter Peterson for their invaluable help in plant care and data collection. We are also grateful to the University of Wyoming Williams Conservatory for access to their facilities. This research was supported by NSF grant \#IOS-1547796.

Abbreviations: Chl $a$-chlorophyll $a$ molecule; Chl $b$ - chlorophyll $b$ molecule; ChlF - chlorophyll $a$ fluorescence; $\mathrm{F}_{\mathrm{m}}{ }^{\prime}-$ maximal fluorescence yield of the light-acclimated state; $\mathrm{F}_{\mathrm{s}}$ - steady-state fluorescence yield; PPFD - photosynthetic photon flux density; RChlF computed value of fluorescence from FluorCAM; $\Phi_{\mathrm{PSII}}$ - effective quantum yield of PSII photochemistry; $\Psi_{\mathrm{L}}$ - leaf water potential. Conflict of interest: The authors declare that they have no conflict of interest. 
the excitation energy is quickly funneled down an energetic gradient to a reaction center (RC), to avoid the possible formation of harmful reactive oxygen species (ROS). Each RC is a distinct proteic structure holding the first $\mathrm{e}^{-}$ donor in the electron transport chain, always represented by Chl $a$. Then, one $\mathrm{e}^{-}$at a time is ejected by Chl $a$ to the primary acceptors to fuel the photosynthetic process or to be dissipated as heat or fluorescence (Butler 1978). Such a prompt, sustained response of both photosystems to light exposure is due to the exceptionally specific atomic arrangement of all molecules taking part in the process. From proximity to the number of chemical bonds and the orientation of each molecule, the LHCs' core is optimized to augment the quantum yield of photosynthesis (Vasil'ev et al. 2004).

Chlorophylls have all a polar 'head' and a nonpolar 'tail' that allows them to associate with chlorophyllbinding proteins in the thylakoid membranes and to interface with the similarly amphipathic phospholipid bilayer (Barber et al. 2000, Lide 2009). The head portion is a hydrogenated porphyrin ring (chlorin) with unshared electron pairs on the oxygen and nitrogen atoms providing polarity. The alternation of single and double bonds in the ring causes a large cloud of resonating electrons, held in place by a chelated magnesium atom, able to lose or gain $\mathrm{e}^{-}$because of multiple valence states. These $\mathrm{e}^{-}$of the ring get instantly excited during the light-harvesting process for all chlorophylls. Although Chl $a$ is an amphipathic molecule just as $\mathrm{Chl} b$, the presence of a methyl $\left(\mathrm{CH}_{3}\right)$ instead of an aldehyde (CHO) in one of its lateral chains, makes it slightly less polar than $\mathrm{Chl} b$. This characteristic causes Chl $a$ to be the endmost molecule in the energy trap within each LHC and makes it the exclusive pigment of the RCs (Battersby 2000, Papageorgiou and Govindjee 2004). When it comes to the three-dimensional structure of the thylakoid membranes in the chloroplast, Chl $a$ is always found to be anchored to lipids or proteins by its hydrophobic tail, leaving the head exposed as an accessible target for excitation energy. This consistent orientation warrants Chl $a$ to be also within easy reach of molecular water highly associated with the photosystem II (PSII) complex (Mirkovic et al. 2017, Saito et al. 2020).

Both photosystem I (PSI) and PSII release chlorophyll $a$ fluorescence $(\mathrm{ChlF})$, with the amount varying with environmental and experimental conditions (Schansker et al. 2005). In the current study, we will focus on the relationship between Chl $a$ and molecular waters in PSII; assuming that the use of short saturating flashes fully reduces the RCs of PSII and ChlF from PSI is negligible (Kalaji et al. 2017). Studies of the PSII crystal structure have made it clear that a high number of water molecules are stably coordinated in well-defined locations of the supercomplex suggesting a functional role for water in PSII beyond the well-characterized photolytic splitting in the oxygenevolving complex. In Thermosynecoccus, seven of the 35 chlorophylls visible in the PSII crystallized structure show persistent ligands with molecular waters and have been proposed to be a critical dynamic proton pathway within the complex (Linke and Ho 2014). Despite growing interest in the possible role of water for PSII as an integral part of the protein and for the enzyme function, specific information on the mechanistic significance of water for the behavior of $\mathrm{Chl} a$ remains scarce. Computational simulations have shown that each PSII is surrounded by $\sim$ 300,000 water molecules, more (bound waters) or less (unbound waters) tightly associated with the complex and almost all of them still have an unknown role (Shen 2015, Vogt et al. 2015). Spectroscopic work has also shown that Chl $a$ can coordinate with two water molecules in hydrophobic media and that hydrogen bonds represent the chemical bridge between two Chl $a$ heads forming RC in at least one of the two photosystems (Shipman et al. 1976, van Bezouwen et al. 2017).

Because ChlF is the result of the energy partitioning within Chl $a$ molecules embedded in the RCs of PSII, its unambiguous signal might be affected by the hydration level within and around this protein-pigment assembly (Vredenberg 2000). During the last few decades, several mechanistic connections between ChlF and leaf photosynthetic activity have been clarified (Genty et al. 1989, Govindjee 2004, Baker 2008, Urban et al. 2017). A substantial number of studies have also described ChlF as a robust signature of plant vigor, able to provide a window into real-time plant stress response under a changing environment (Lichtenthaler 1998, Maxwell and Johnson 2000, Baker and Rosenqvist 2004, Woo et al. 2008, Murchie and Lawson 2013, Kalaji et al. 2016, Begović et al. 2020). In particular, both Pulse Amplitude Method (PAM) traces and images of ChlF have found enormous use in research focusing on plant response to water limitations, with successful results across species and life stages, from early sensing of drought to droughtinduced mortality (Lichtenthaler and Miehé 1997, Yao et al. 2018, Gu et al. 2019, Guadagno and Ewers 2020). Still, the changes in ChlF under water limitation lack fully mechanistic explanations; more specifically, the role of PSII's molecular water and its microenvironmental dynamics remain unexplained.

We used a progressive drought treatment to quantify how variation in physiological traits thought to be informative of the overall leaf hydration (e.g., LWC - leaf water content, $\Psi_{\mathrm{L}}$ - leaf water potential) (Scoffoni et al. 2014), influence ChlF per image. Since ChlF images were captured as single screenshots from fresh-cut plant material using an enclosed camera (FluorCAM - Photon Systems Instruments, Drásov, Czech Republic), we first developed a pipeline to compute a relative $\mathrm{Chl}$ fluorescence (RChlF) value based on pixel counts associated with red, green, and blue bands for each image (Eq. 1). After comparing these results with in vivo data obtained using a handheld multispectrophotometer (Multispeq - PhotosynQ LLC, East Lansing, MI), we tested how RChlF varied with hydration across species with morphologically extreme leaf types. Specifically, we studied the response of leaves from two morphotypes in the species Brassica rapa, an oilseed and a turnip, and from a widespread, North American tree, Populus tremuloides. We compared this broad-leaved tree and crops to needles from Pinus ponderosa and to the 
small leaves of sagebrush (Artemisia tridentata), a desert shrub, so to cover an extreme spectrum of leaf morphology, greenness (i.e., absorbance and pigmentation), and secondary features (e.g., presence of hairs, thorns, oilproducing glands). To explain the dependency of the ChlF signal upon leaf hydration, we hypothesize a mechanistic model where the conformational stability and function of the Chl $a$ molecules in PSII is controlled by changes in the pool of waters in the complex's microenvironment and weakens under stress conditions.

\section{Materials and methods}

Plant material: In the spring of 2018, a progressive drought experiment was conducted on plants grown in 4.8-L pots in a glasshouse environment. Two-year-old aspen ( $P$. tremuloides) and pine ( $P$. ponderosa) seedlings were purchased from the Montana Conservation Nursery and the University of Idaho Nursery, respectively. Both species were grown using sieved, native soils from the Medicine Bow National Forest in Wyoming $\left(41^{\circ} 14^{\prime} 49.0^{\prime \prime} \mathrm{N}, 105^{\circ} 26^{\prime} 53^{\prime \prime} \mathrm{W}\right)$. The same soil was used to plant two-year-old seedlings of big sagebrush, sage (A. tridentata), from the New Mexico Forestry Division. All species were fully (i.e., at soil capacity) watered for three weeks after potting to allow for proper establishment. Two B. rapa morphotypes (Ashraf and Mehmood 1990, Lin et al. 2014) were also grown to implement the diversity of the experimental panel. In-house bulked seeds (University of Wyoming, 2012) were used for the oilseed (R500, B. rapa ssp. trilocularis, Yellow Sarson), while seeds for the turnip type (VT-089, D'Auvergne Hative) were purchased from the Wageningen University and Research Center for Genetic Resources (CGN\#10995). For both $B$. rapa types, seeds were first planted in a soil mixture [Miracle-Gro moisture control Potting Mix, 20\% v/v, Marysville, $\mathrm{OH}$, and Profile Porous Ceramic (PPC) Greens Grade (80\% v/v), Buffalo Grove, IL] amended with $4 \mathrm{ml}$ of Osmocote 16-6-12 fertilizer (Scotts, Marysville, $\mathrm{OH})$ in $500-\mathrm{mL}$ pots. After four weeks of daily watering, de-potted $B$. rapa plants were moved to 4.8 -L pots with the addition of $\sim 4.0$ L of sand (Premium Play Sand, Quickrete, Atlanta, GA).

Environmental conditions and design: The greenhouse photoperiod was extended to $12 \mathrm{~h}$ total by adding LED lights for $2 \mathrm{~h}$ (Lumigrow, Emeryville, CA) at dawn $(\sim 5: 30 \mathrm{~h})$ and dusk $(\sim 17: 10 \mathrm{~h})$ for the duration of the experiment. Air temperature ranged between $25-37^{\circ} \mathrm{C} /$ $18-22^{\circ} \mathrm{C}$ (day/night) while average PPFD during the day ( \pm standard deviation) was $600 \pm 200 \mu$ mol(photon) $\mathrm{m}^{-2} \mathrm{~s}^{-1}$, measured with a sensor posed at average plant height (LI190SB, LiCor Inc., Lincoln, NE). Soil volumetric water content (VWC) was monitored with Echo5 probes (Decagon Devices Inc., Pullman, WA, USA) calibrated to each soil using gravimetric water content (Campbell et al. 2007). Gypsum blocks were used for estimating soil water potential (SWP) and calibrated using psychrometric procedures (WP4-C, Decagon Devices Inc., Pullman, WA, USA). All environmental and soil data were recorded at 15-min intervals on CR3000 data loggers (Campbell Scientific Inc., Logan, UT). A total of 150 plants $(n=30$ replications in each species) were spaced across the greenhouse and at least six replicates per species for droughted and fully watered (controls) plants were randomly selected for measurements over $52 \mathrm{~d}$ of progressive drought as reported in Beverly et al. (2020).

In vivo ChIF measurements and related physiological traits: Over the course of $52 \mathrm{~d}$ from the start of the drought, at each time point (once or twice a week based on drought progression) between 10 and 12 (5-6 wellwatered and 5-6 droughted) replicate plants of each species were randomly selected and measured with the MultispeQ spectrophotometer (PhotosynQ LLC, East Lansing, MI). Due to their very different morphological characteristics (Table 1), some species-specific alterations to the procedure were applied. In Table 1, the foremost differences across the experimental panel are reported. Leaf thickness, measured in vivo with the MultispeQ, is reported for reference across species as for leaves in wellwatered conditions. All other features are qualitatively expressed, representing the result of visual inspections and supporting literature information (Anderson et al. 2011, Lin et al. 2014, Chitwood and Sinha 2016).

All measurements were taken on the youngest fully expanded leaves for the $B$. rapa while one leaf from the most healthy lateral shoot was used for each measurement of sage. For aspen, one healthy leaf from the high-mid part of each plant was measured while nine needles for each pine were placed as flat as possible in the instrument's clamp to ensure full coverage of the measuring surface and to avoid light spills. These in vivo ChlF measurements were taken always between 11:00 and 13:00 h mountain standard time (MST) and the protocol is available on the PhotosynQ platform under project title: PABST Conservatory 2018 (https://photosynq.org/projects/pabst_conservatory-2018). In a single 2-min clamping, the protocol measured several ChlF-related parameters, including $\mathrm{F}_{\mathrm{m}}{ }^{\prime}$ - maximal fluorescence yield of the light-acclimated state, $F_{s}-$ steady-state fluorescence yield, $F_{v}{ }^{\prime} / F_{m}{ }^{\prime}-$ theoretical efficiency of PSII in the light-acclimated state, $\Phi_{\text {PSII }}$ - effective quantum yield of PSII photochemistry, and LEF - linear electron transport rate (Tietz et al. 2017). Saturating pulses were maintained higher than 3,000 $\mu$ mol(photon) $\mathrm{m}^{-2} \mathrm{~s}^{-1}$ for all measurements with $0.8-\mathrm{s}$ duration and $\lambda=470 \mathrm{~nm}$. The Multispe $Q$ also collected SPAD - relative chlorophyll content and absorbance at $450,535,605,650,730,850,880$, and $940 \mathrm{~nm}$. Moreover, leaf thickness (in $\mathrm{mm}$ ) along with leaf temperature and environmental conditions (PPFD and ambient temperature/pressure/humidity) were synchronously tracked and a full list of all Multispe $Q$ recorded data can be found at https://github.com/crguadagno/ChlF-hydration-2020.

ChlF images collection and analysis: At each time point, ChlF images were captured between 11:00 and 13:00 h (MST) to avoid inconsistent lighting conditions between the in vivo measurements and the imaging. The same leaves for both the Multispe $Q$ measurements and the 
Table 1. Summary of the foremost differences across the experimental panel. Leaf thickness, measured in vivo with the MultispeQ, is reported for leaves in control (i.e., fully watered) conditions. All other features are qualitatively expressed, representing the result of visual inspections and supporting literature information (Anderson et al. 2011, Lin et al. 2014, Chitwood and Sinha 2016).

\begin{tabular}{lllll}
\hline & &
\end{tabular}

imaging work for each replicate plant were selected. When the original leaf was somewhat damaged during the in vivo measurement, another leaf or needles were chosen in the same cohort for health and age from the same replicate plant. Leaves and needles were placed flat onto the imaging plate of a closed FluorCAM (FC 800-C, Photon Systems Instruments, Drásov, Czech Republic) for the imaging protocol. This consisted of a series of screenshots before $\left(\mathrm{F}_{\mathrm{s}}\right)$ and immediately after $\left(\mathrm{F}_{\mathrm{m}}{ }^{\prime}\right)$ the application of a 0.8 -s saturating pulse $\left[\sim 4,000 \mu\right.$ mol(photon) $\left.\mathrm{m}^{-2} \mathrm{~s}^{-1}\right]$ using an actinic light of $\sim 800 \mu \mathrm{mol}$ (photon) $\mathrm{m}^{-2} \mathrm{~s}^{-1}$ and $\lambda=470 \mathrm{~nm}$ used to match the average PPFD across canopy in the greenhouse at that time of day. Using $R$ (version 3.6.3), we developed a computing pipeline for image analysis ('raster' and 'sf' $R$ packages) to select for regions of different ChlF intensity. Pixels for individual red, green, and blue color bands were outlined using three layers for image colors, and pixels in each layer were then counted. Band assignment was dictated by the definition of minimal ChlF from leaves in dark-acclimated conditions and pulsing red baseline light probe in the FluorCAM. Maintaining consistent saturation settings, equal distance between camera and samples, and times of measurements enabled us to compare and contrast images from diverse samples throughout the progressive drought experiment. Specifically, pixels of the red regions were defined as highly fluorescing $\left(R_{\text {pix }}\right)$, green pixels $\left(G_{\text {pix }}\right)$ as moderately fluorescing, and blue pixels as the ones with baseline ChlF levels $\left(\mathrm{B}_{\mathrm{pix}}\right)$. The computed values for relative chlorophyll fluorescence (RChlF) were next calculated according to Eq. 1:

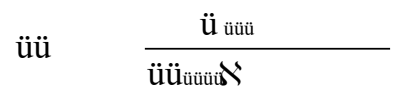

where actively fluorescing pixels $\left(R_{\text {pix }}\right.$ and $\left.G_{\text {pix }}\right)$ are compared to the sum of all pixels in the image. ChlF images for the presented analyses are available from the University of Wyoming data repository (https://mountainscholar.org/) and the source files and code are available at https://github. com/dbeverly/ImagingWaterStatus.

Leaf hydration measurements: At each time point, after the ChlF images were collected, all leaves and needles were inserted into a Scholander pressure chamber (model 600 Pressure Chamber Instrument - PMS Instrument; Koide et al. 1989) to estimate leaf water potential $\left(\Psi_{\mathrm{L}}\right)$. After all measurements, leaves and needles were saved for biomass determination. Fresh $\left(\mathrm{FM}_{\text {leaf }}\right)$ and dry leaf mass $\left(\mathrm{DM}_{\text {leaf }}\right)$ were determined on harvesting days and after drying at $65^{\circ} \mathrm{C}$ at least $10 \mathrm{~d}$, respectively. Water content (WC) was then calculated by subtracting dry leaf mass from the fresh leaf mass for each sample. Leaf water content (LWC) values were normalized to initial fresh mass according to Eq. 2:

$$
\text { ü } \quad \frac{\text { üü üüüü }}{\text { ü } \text { üü }}
$$

For pine and sage, species less likely to lose water during the time between harvesting and ChlF images recording, we also calculated relative water content (RWC) according to Eq. 3:

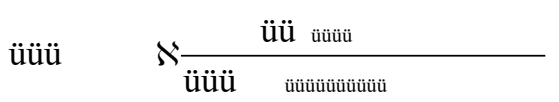

substituting the saturated mass values (i.e., at turgor) with species-specific averages of the fresh mass values recorded 
for fully watered samples (i.e., controls) at predawn (Weatherley 1950).

Statistical analyses: The direct comparison of RChlF and different ChlF-related parameters across all species was performed using a series of parametric correlation tests (Pearson's) using $R$ - version 3.6.3 to verify linear dependence between each pair of variables. The linear model in $R$ for $\mathrm{VWC}, \Psi_{\mathrm{L}}$, and LWC relative to imaging performance (RChlF), used 146 independently measured plant values across all species over $52 \mathrm{~d}$ of progressive drought. The linear model for RWC was instead set in $R$ selecting for 20 independently measured sage and pine values across all levels of soil moisture. All data and codes are available at https://github.com/crguadagno/ChlFhydration-2020.

\section{Results and discussion}

We began the process of testing a molecular mechanism for hydration impacts on PSII function through an experimental panel of diverse plant leaf morphotypes (Fig. 1) exposed to water dynamics from well-watered to lethal drought. ChlF quantified from images is commonly utilized to monitor the activity of PSII across species and environmental settings (Gorbe and Calatayud 2012). However, PAM protocols can be lengthy and comparisons between hand-held and imaging fluorometers are still scarce (Guadagno and Ewers 2020, Herritt et al. 2020). Experiments involving diverse species are also uncommon limiting our understanding of the mechanisms behind the reflection of photosynthetic efficiency in ChlF dynamics. We developed a rapid pipeline to analyze ChlF images that we validated against in vivo measurements (Fig. 2). Extremely different leaf characteristics in our experimental panel (Fig. 1, Table 1) seemed to explain species-specific variations for samples at the highest hydration level (Fig. 3). We then explored the relationship between the signal detected via imaging ChlF and tissue hydration during progressive drought (Figs. 4, 5) with results suggestive of a mechanistic model driven by the structural interaction between Chl $a$ molecules and waters occurring in and around PSII (Fig. 6).

RChIF from images and ChlF in vivo: Despite its current wide range of applications (Murchie and Lawson 2013, Kalaji et al. 2016), imaging ChlF has been criticized in the past for its constricted relevance (i.e., one band in one wavelength region) in answering ecophysiological questions (Lichtenthaler et al. 2005). In theory, PAM measurements would only represent the efficiency of the uppermost chloroplast layer of the leaves' palisade cells, especially in very dark green leaves (Lichtenthaler and Miehé 1997). Moreover, the impact of hydration level and morphological leaf characteristics $\mathrm{ChlF}$ imaging products is still unclear (Murchie and Lawson 2013).

To investigate the possible effects of leaf hydration on ChlF images, we followed plants naturally dried through a complete water withholding treatment for consecutive $52 \mathrm{~d}$ in controlled greenhouse conditions (Beverly et al. 2020). Our experimental panel included species with extremely different leaf characteristics and diverse drought susceptibility: two crop types in the species $B$. rapa, two common North American woody species, and a shrub (Table 1). This panel provided a critical comparison of ChlF imaging performance in broad leaves (oilseed, turnip, aspen), small leaves (sage), and needles (pine) (Fig. 1F). Both the images' capture and the consequent computational analysis can be impacted by obvious differences in leaf morphology (e.g., shape, average size, veins' width, thickness), but also by species-specific greenness, a result of absorbance and pigmentation, and by less evident secondary features (e.g., presence of hairs, thorns, oil-producing glands) (Table 1$)$. The $B$. rapa crop types are the results of years of domestication (Lin et al. 2014) leading to moderately thin, opposite leaves and a short life cycle for the oilseed, which flowers relatively early ( 7 weeks) and produces hundreds of seeds per plant (Fig. 1A). In contrast, the turnip type is slow growing, with flowers that only appear if induced by a vernalization

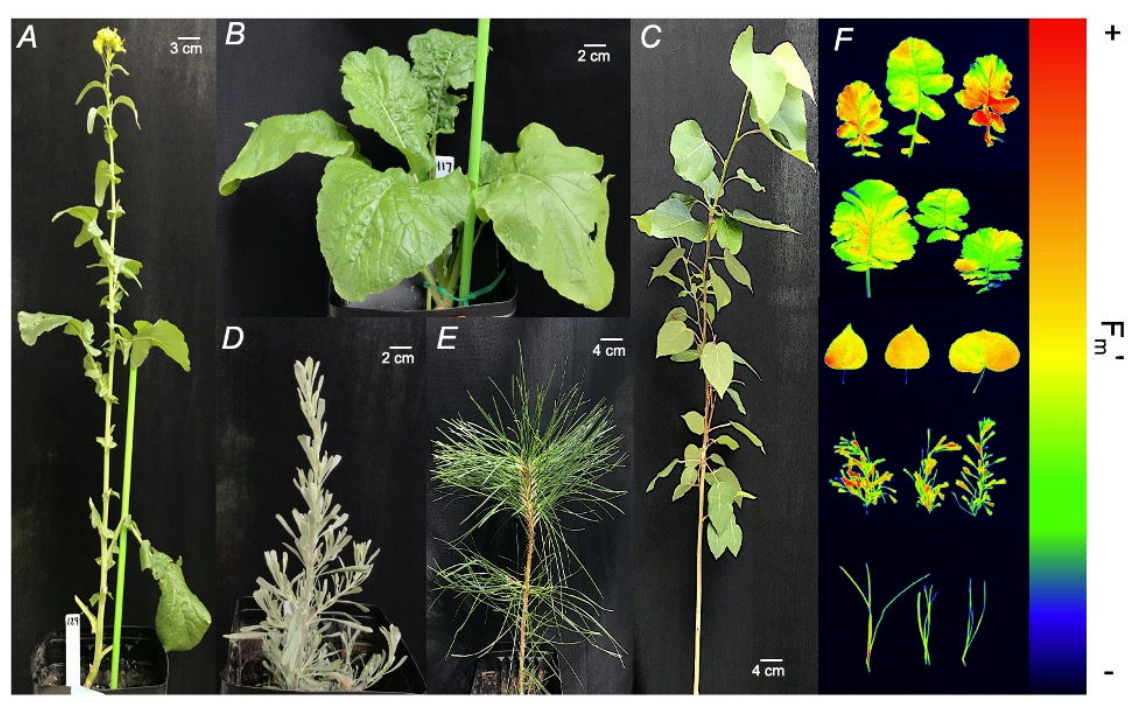

Fig. 1. In vivo pictures of the experimental panel $(A-E)$ and $\mathrm{ChlF}$ images from fresh-cut leaves from each species $(F)$. Fully hydrated potted plants of Brassica rapa - oilseed (A), Brassica rapa - turnip (B), Populus tremuloides aspen $(C)$, Artemisia tridentata - sage (C), and Pinus ponderosa - pine (E) are represented. ChlF images were captured after a saturating pulse at the FluorCAM; false color scale indicating $F_{m}$ ' value as the maximal fluorescence yield of the light-acclimated state, with oilseed, turnip, aspen, sage, and pine reported from top to bottom $(F)$. 
period, and the presence of a storage organ (i.e., turnip) which started developing in potted plants at $\sim 4$ weeks from germination. The leaves are thorny in the turnip type, and they are arranged in a rosette display (Fig. $1 B$ ). The other two considered angiosperms, aspen and sagebrush, have leaves that adapted to the conditions of their native environments (Anderson et al. 2011, Chitwood and Sinha 2016). Thin but very firm leaves characterize the deciduous tree, while hairy, thick but very small leaves are typical of the xeric shrub (Fig. 1C,D). We also added a gymnosperm to our experimental panel, Pinus ponderosa (Fig. 1E), characterized by dark olive-green long needles assembled in threes, with a thick cuticle, covered by a waxy layer to prevent water loss (Conkle and Critchfield 1988).

At each time point during the course of the progressive drought, we captured images of leaves and needles with the FluorCAM before and immediately after the application of a saturating pulse. Since all leaves were in light-acclimated conditions, we expected for all measurements at the FluorCAM, the first image to be informative of ChlF yield at steady-state $\left(\mathrm{F}_{\mathrm{s}}\right)$, while the second to be comparable with the maximal yield of $\mathrm{ChlF}\left(\mathrm{F}_{\mathrm{m}}{ }^{\prime}\right)$ of a typical PAM protocol (Brooks and Niyogi 2011). Using Eq. 1, we obtained RChlF values for both conditions, pre- and post-saturating pulse, and tested the relationship between RChlF and different ChlF variables recorded with the MultispeQ (in vivo). By the end of the experiment, droughted plants across all species reached mortality, and this was reflected in the near-zero and zero values for ChlF that were recorded for both imaging and handheld methods (Fig. 2). The progressive drought treatment indeed led to remarkably low values of soil moisture (SM [\%]), unsustainable even for a xeric species like sage when grown in pots (Fig. 1S, supplement). When comparing the computed $\mathrm{RChlF}$ to MultispeQ results, the relations were poor when we used pre-pulse $\mathrm{RChlF}$ (Fig. 2S, supplement) but they significantly improved when the post-pulse values for RChlF were considered (Fig. 2). The Multispe $Q$ values for quantum yield of PSII, $\Phi_{\text {PSII }}$ (Fig. 2A), $\mathrm{F}_{\mathrm{v}}{ }^{\prime} / \mathrm{F}_{\mathrm{m}}{ }^{\prime}$ (Fig. 2B), or $\mathrm{F}_{\mathrm{s}}$ (Fig. 2C), did not show a high significant correlation to post-pulse RChlF values. However, a highly significant linear dependency $(R=0.88)$ emerged when RChlF was compared to $\mathrm{F}_{\mathrm{m}}{ }^{\prime}$ (Fig. 2D). This is partly because $\Phi_{\text {PSII }}$ and $\mathrm{F}_{\mathrm{v}}{ }^{\prime} / \mathrm{F}_{\mathrm{m}}{ }^{\prime}$ are directly affected by the efficiency of the downstream LEF while $F_{\mathrm{m}}{ }^{\prime}$ simply reflects the compartmentalized response of the RCs in PSII to the saturating pulse. It has been indicated before that the reduction capability per RC has low variability in healthy leaves and that ChlF values in light conditions are meaningful of stress response from early sensing to mortality (Maxwell and Johnson 2000, Demmig-Adams et al. 2014a, Guadagno et al. 2018). Here we showed that $F_{m}{ }^{\prime}$ is a more solid variable than $F_{s}$ in a multispecies evaluation and that the underpinning consistency in $\mathrm{RCs}^{\prime}$ reduction rates holds true under extreme stress conditions. However, the correlation between RChlF and $\mathrm{F}_{\mathrm{m}}{ }^{\prime}$ was not maximal because the images were captured on cut leaves while the Multispe $Q$ was used for in vivo measurements causing a time lag between the two procedures. These encouraging results revealed the RChlF original pipeline for FluorCAM products to be dependable when used across a wide range of VWC $(1.4 \% \leq \mathrm{VWC} \leq 26.4 \%)$, evaluated across diverse species. Noteworthy, the choice of taking only two screenshots pre- and post-pulse instead of a full PAM protocol for a fluorescence curve decreased the image acquisition time from minutes to seconds with immense improvement in the final throughput.

When analyzing results from the control plants at the highest hydration level $(\mathrm{SM} \leq 26 \%)$, which were

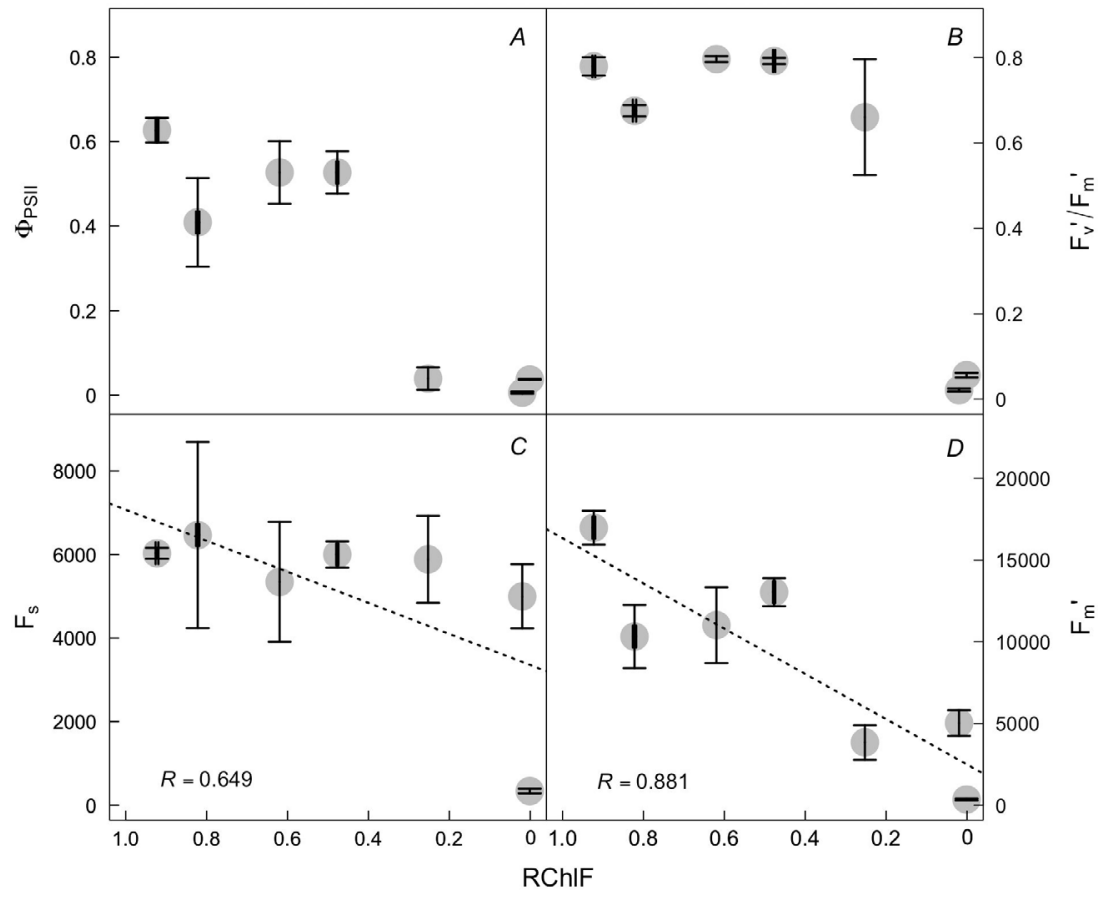

Fig. 2. Comparison of computed RChlF (Eq. 1) from the FluorCAM images (postpulse) and in vivo ChlF-related traits from the MultispeQ. $\Phi_{\mathrm{PSII}}$ - the effective quantum yield of PSII photochemistry (A), $\mathrm{F}_{\mathrm{v}}{ }^{\prime} / \mathrm{F}_{\mathrm{m}}{ }^{\prime}$ - theoretical efficiency of PSII in the light-acclimated state $(B)$, $\mathrm{F}_{\mathrm{s}}$ - the steady-state fluorescence yield (C), and $\mathrm{F}_{\mathrm{m}}{ }^{\prime}$ - the maximal fluorescence yield of the light-acclimated state $(D)$ are represented. Gray dots are the average values (total $n=332$ measurements) across all species (oilseed, turnip, aspen, sage, and pine) over $52 \mathrm{~d}$ of progressive drought with the error bars (SE). Dotted lines show the correlation between RChlF and $\mathrm{F}_{\mathrm{s}}(C)$ or $\mathrm{F}_{\mathrm{m}}{ }^{\prime}(D) . R$ values are results of Pearson's correlation test. 
never exposed to drought during the experiment, speciesspecific features directed the range of variation in $\mathrm{RChlF}$ (Fig. 3). For these fully hydrated samples, both oilseed and turnip $B$. rapa grouped with the aspen maintaining a RChlF always higher than 0.75 , while pine and sage showed lower values between 0.45 and 0.72 . Searching for possible drivers of RChlF across species, we found these trends consistent with relative chlorophyll content (CC) (Fig. 3A), leaf thickness (Fig. 3B), SPAD at $530 \mathrm{~nm}$, and absorbance at $850 \mathrm{~nm}$ (Fig. 3S, supplement) from the MultispeQ. All in vivo measurements had an actinic light ranging $500 \mu \mathrm{mol}$ (photon) $\mathrm{m}^{-2} \mathrm{~s}^{-1} \leq$ PPFD $\leq 1,600$ $\mu \mathrm{mol}$ (photon) $\mathrm{m}^{-2} \mathrm{~s}^{-1}$ that did not seem to affect the speciesspecific distribution of the data (Fig. 4S, supplement). The species-specific variation of RChlF in the fully hydrated samples likely corresponds to the significant difference in leaf thickness of aspen $(0.44 \pm 0.04 \mathrm{~mm})$ and both $B$. rapa genotypes $(0.6 \pm 0.06 \mathrm{~mm})$ when compared to sage $(1.9 \pm 0.5 \mathrm{~mm})$ and pine $(1.6 \pm 0.52 \mathrm{~mm})$ (Fig. $3 B)$. The Multispe $Q$ records $\mathrm{CC}$ as $100 \times \log$ (transmittance at $940 \mathrm{~nm} /$ transmittance at $650 \mathrm{~nm}$ ), expressed per unit area, which thus integrates the chlorophyll content per unit mass and leaf thickness (Kuhlgert et al. 2016). Secondary features, such as the waxy layer of pine needles and the hairy surfaces characterizing sage leaves, additionally limited these species in reaching the highest levels of $\mathrm{RChlF}$ since they impact the absorbance signature of leaves (Table 1). Leaf characteristics for sage and pine derive from a leaf life span greater than one growing season so they come with tradeoffs of hardiness vs. light harvesting and overall photosynthetic capacity (Wright et al. 2004). Past calibration equations for the SPAD measurements were usually species-specific reflecting traits such as leaf thickness and leaf succulence (Richardson et al. 2002, Uddling et al. 2007, Fernández-Calleja et al. 2020). Our study indicates that leaf thickness and secondary leaf characteristics (Table 1) lead to species-specific behavior in RChlF in fully hydrated samples, but that species variation decreases when hydration decreases (Fig. 2D). Thus, RChlF is not solely a composite of speciesspecific leaf characteristics, but it is also the result of fundamental biophysical molecular mechanisms that can be standardized across species (Baker 2008). We suggest that the relationship between Chl $a$ and the PSII hydration layer mechanistically explains these widespread processes and these molecular dynamics within the membrane become a limiting factor for ChlF at decreasing hydration levels. Future experiments that couple leaf-level physiology measurements to computational tools used to study the impact of structural alterations to biological systems (e.g., molecular dynamics) will likely help testing this hypothesis across scales (Porcar-Castell et al. 2014, Magney et al. 2020).

RChIF and means of hydration under progressive drought: Imaging ChlF has been successfully utilized in the past to quantify the variation in PSII efficiency under decreasing water availability, eventually leading to cellular membrane failure (Woo et al. 2008, Guadagno et al. 2017). Previous work has underlined the relevance of leaf succulence in considering SPAD and absorbance (Campbell et al. 1990, Marenco et al. 2009). In these studies, leaf succulence was correlated to more or less direct traits, such as chlorophylls' production and nitrogen content. Our work makes progress by analyzing the impact of changes in hydration (i.e., a means of leaf succulence)
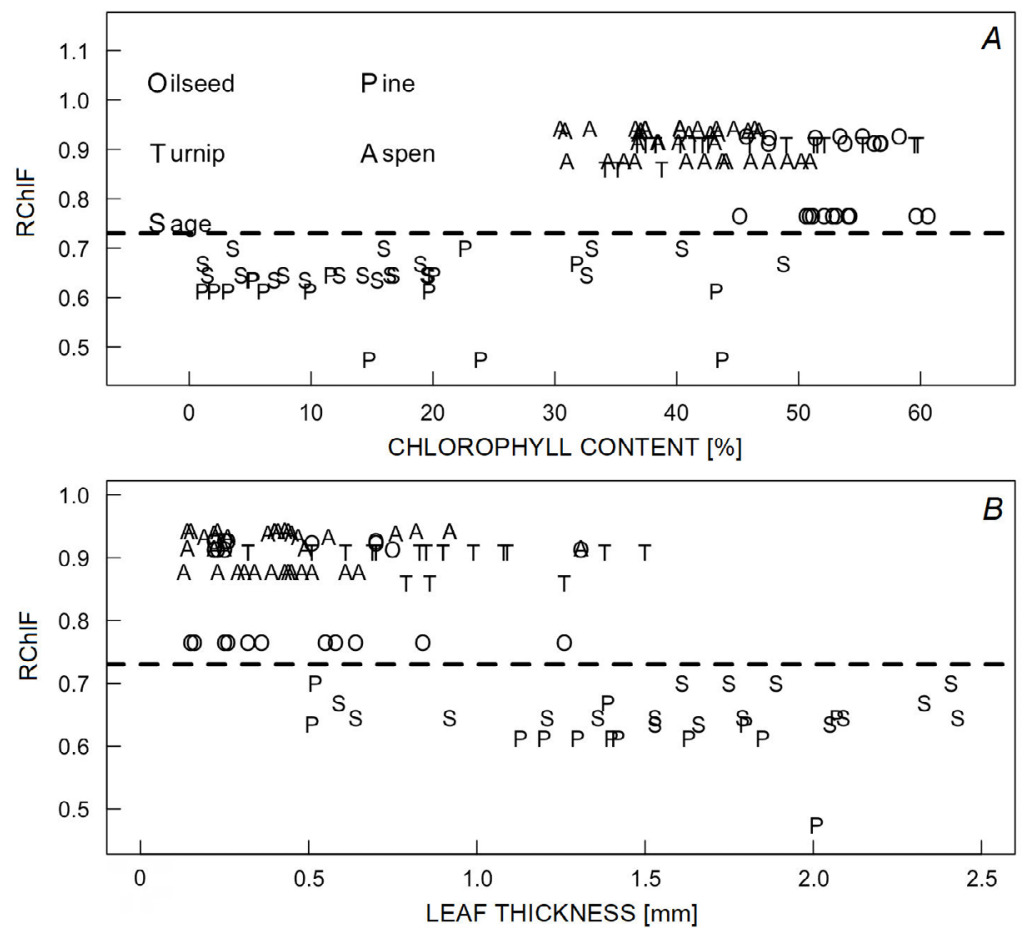

Fig. 3. Variation of computed RChlF (Eq. 1) from the FluorCAM images (post-pulse) with Multispe $Q$ traits across species at full watering. Single measurement for oilseed, turnip, aspen, sage, and pine are denoted by capital letters: $\mathrm{O}, \mathrm{T}, \mathrm{A}, \mathrm{S}$, and $\mathrm{P}$, respectively. Variation with chlorophyll content as a percentage $(A)$ and thickness in $\mathrm{mm}(B)$ are reported. Dotted lines represent the separation of species into two groups based on the range of RChlF recorded for control well-watered leaves or needles. 
on ChlF per image to provide a more mechanistic model where Chl $a$ 's molecular behavior, more than its content or accumulation, determines the trait's dynamics.

Our experiment resulted in the death of each plant in the droughted cohort across all species: the $B$. rapa crop types died after $21 \mathrm{~d}$ of continuous water withholding (minimal VWC $<7.2 \%$ ), aspen (VWC $<6 \%$ ) and pine (VWC $<6.3 \%$ ) after $45 \mathrm{~d}$, and sage died at a minimal VWC $<5.1 \%$, after $52 \mathrm{~d}$ in drought. Mortality, assessed as the lack of meristematic recovery upon full rewatering for two weeks, progressed according to the expected speciesspecific drought sensitivity, driven by the rate of water use and lethal water potential thresholds, even though all plants were growing in pots and in greenhouse conditions (Table 1). We tested different hydration-related traits to find the one with the highest potential mechanistic value, determined as correlation coefficient, in explaining drought-induced variations in RChlF (Figs. 4, 5) and
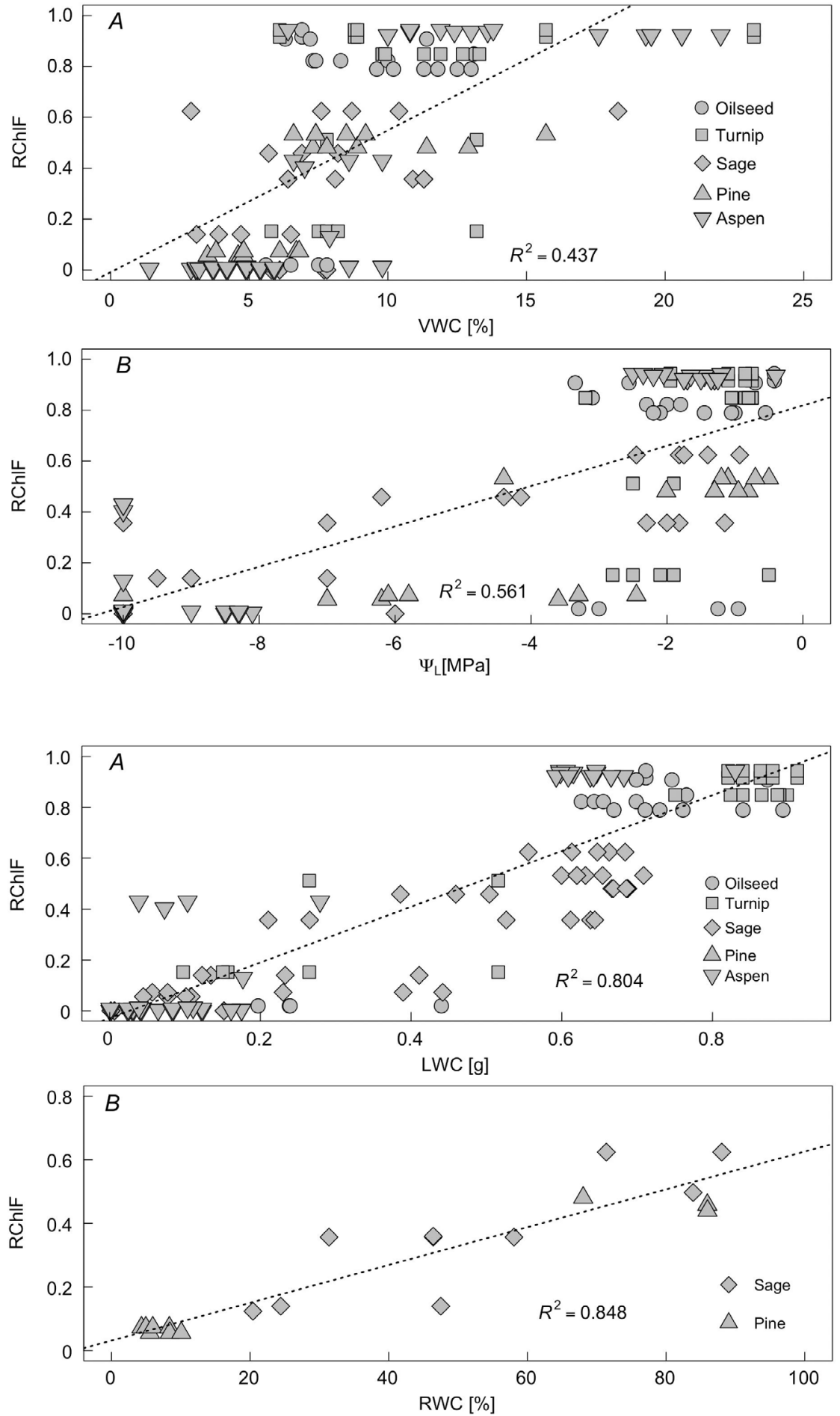

Fig. 4. Variation of computed $\mathrm{RChlF}$ (Eq. 1) from the FluorCAM images (post-pulse) with means of hydration across species under progressive drought. Single measurement for varying RChlF at changing volumetric soil water content (VWC) as a percentage $(A)$ and leaf water potential $(B)$ are reported. A linear model for VWC considering all species (dotted line) results in $\mathrm{x}=-0.0316027+0.0059418 \mathrm{y}$ with $R^{2}=0.44$ and $p<0.005$, while a model for $\Psi_{\mathrm{L}}$ in $\mathrm{x}=-0.008898+$ $0.055755 \mathrm{y}$ with $R^{2}=0.56$ and $p<0.005$.

Fig. 5. Variation of computed RChlF (Eq. 1) from the FluorCAM images (post-pulse) with means of hydration across species under progressive drought. Single measurement for varying RChlF at changing leaf water content (LWC) in grams across all species $(A)$ and relative water content (RWC) for only sage and pine $(B)$ are reported. LWC and RWC were calculated according to Eq. 2 and 3, respectively. A linear model for LWC considering all species (dotted line) results in $\mathrm{x}=-0.02896+1.09529 \mathrm{y}$ with $R^{2}=0.80$ and $p<0.005$, while a model for RWC in $\mathrm{x}=-0.0316027+$ $0.0059418 \mathrm{y}$ with $R^{2}=0.85$ and $p<0.005$. 
RChlF computed from post-pulse images, $\mathrm{F}_{\mathrm{m}}{ }^{\prime}$ (Fig. 1F). Although RChlF varied linearly with VWC and $\Psi_{\mathrm{L}}$ within each of the considered species, we found low correlation when verifying its direct dependence across all species (Fig. 4). On the contrary, using the same 146 independently measured plants across all species, RChlF showed a highly significant $\left(R^{2}=0.80\right)$ linear correlation with leaf water content, LWC (Fig. 5A). Our results are in line with earlier evidence for LWC to be a crucial driver of plant spectral signature in both field and greenhouse conditions (Campbell et al. 1990, Marenco et al. 2009, Murphy et al. 2019, Sun et al. 2019).

The use of a diverse experimental panel was indeed advantageous for testing the significance of the correlation, and thus the potential mechanistic explanation, across species with dissimilar leaf characteristics that we usually do not find growing in the same canopy (Table 1). RChlF maintained a robust linear response with decreasing LWC for all droughted plants (Fig. $5 \mathrm{~A}$ ) even if the images were collected on cut leaves and needles creating a time lag in the procedure. This supported the robustness of our pipeline and confirmed our proxy (RChlF) to maintain an overall mechanistic relation with hydration despite the possible procedure's artifact in the measurements. The cut itself and the time spent at the FluorCAM could have in fact unsettled the in vivo leaf water status, with leaves starting to passively leak water from the wound and quick stomata shutdown. This was already implied by the poor predictions of stomatal conductance from RChlF obtained on the same experimental panel (Beverly et al. 2020). So, we tested RChlF against the RWC finding an even higher linear correlation $\left(R^{2}=0.85\right)$ than for LWC (Figs. 4B, 5B). For these calculations (Eq. 3), we used species-specific averages of the fresh mass values recorded for fully watered samples at predawn as mass values at turgor and calculated RWC only for the most xeric species, sage and pine. This increased correlation was indicative of a species-specific response to the time lag imposed by the method; species characterized by high leaf succulence, such as the $B$. rapa plants, are in fact more prone to lose water when detached with respect to pine needles and the hairy leaves of sage (Table 1). All means of hydration we considered showed different possible mechanistic values when it comes to explain RChlF (i.e., computed $\mathrm{F}_{\mathrm{m}}{ }^{\prime}$ ). $\mathrm{RWC}$, for its direct association to the hydration state of the mesophyll cells in the leaf and their ultrastructural changes under stress (Giles et al. 1974, Radermacher et al. 2019), was the most informative trait of the actual PSII hydration status from leaf measurements in our correlation analysis.

Altogether, our results corroborated our premise of the existence of biophysical, process-based relations between hydration and the signal of ChlF from PSII that goes beyond regulated photoprotective mechanisms (Ruban 2016). Given the evidence that ChlF (and its derived variables) is a reliable mirror of water stress impact on PSII efficiency (Lichtenthaler et al. 1986, Baker and Rosenqvist 2004, Woo et al. 2008), we leveraged our multi-species imaging products to introduce a molecular model that uses the structural foundations of the Chl $a$-water relationship in PSII to clarify the mechanisms behind ChlF dynamics under changing hydration levels.

Hypothesized model of interaction for $\mathrm{Chl} a$ and molecular waters impacting ChIF signal: The reduction in PSII efficiency and ChlF signal under stress has been mainly explained through the presence of the multifaceted, protective, and finely regulated mechanism of nonphotochemical quenching of ChlF (NPQ), critical to reduce oxidative stress (Baker 2008, Demmig-Adams et al. 2014b, Ruban 2016). Inactivated PSIIs (i.e., RCs actively absorbing light but unable to conduct photochemistry) have also been shown to maintain their dissipation capacity in vivo through a shorter lifetime of ChlF (Matsubara and Chow 2004). These different safety valves for the photosynthetic machinery all depend on the connectivity of the LHCII, and physical changes in the PSII microenvironment will certainly affect the overall energy partitioning. Due to the elevated numbers of water molecules in and around PSII, we used a hydration experiment across diverse species to explore how fluctuations in hydration levels, observed to change cellular ultrastructures in mesophyll cells (Radermacher et al. 2019), might lead to the eventual disruption of the chemical bonds between water molecules and Chl $a$. The disruptions from lack of molecular hydration around PSII will affect the signal of ChlF even beyond photoinactive PSII (Shen 2015, Vogt et al. 2015, Mirkovic et al. 2017).

Although the role of molecular waters as the substrate of PSII and the partitioning of excitation energy in the RCs is well understood (Govindjee 2004), how waters might also physically impact the ChlF signal from PSII remains unexplored. Recent experimental evidence from crystal structures has confirmed the presence of hundreds of thousands of molecular waters in and around isolated PSIIs with unknown function (Linke and Ho 2014, Shen 2015, Vogt et al. 2015). In a healthy, fully hydrated (i.e., unstressed) PSII complex, both bound and unbound waters are more or less, with higher or lower number of hydrogen bonds, tightly connected to the amino acid residues of this thylakoidal transmembrane protein (Fig. 6A). In this three-dimensional structure, Chl $a$ molecules are anchored to the complex by their hydrophobic tail, very likely through water-soluble chlorophyll proteins (WSCPs; Girr et al. 2020), leaving the head portions of the molecule exposed for excitation energy and light harvesting (Mirkovic et al. 2017, Saito et al. 2020) (Fig. 6A-inset). This stringent orientation for Chl $a$ in the structure is dictated by its amphipathic nature, and we propose that molecular waters are key for the pigment to interact efficiently with $\mathrm{e}^{-}$and amino acid residues of PSII, resulting in the highest ChlF signal (Fig. 6A). Possible interactions between Chl $a$ and water in PSII have been suggested in the past without further analysis of the impact on ChlF (Shipman et al. 1976, Agostiano et al. 2002, van Bezouwen et al. 2017). In our model, with decreasing leaf hydration, changes in the chloroplastic water potential will cause the unbound waters in the PSII's microenvironment to leave the structure, diminishing the 

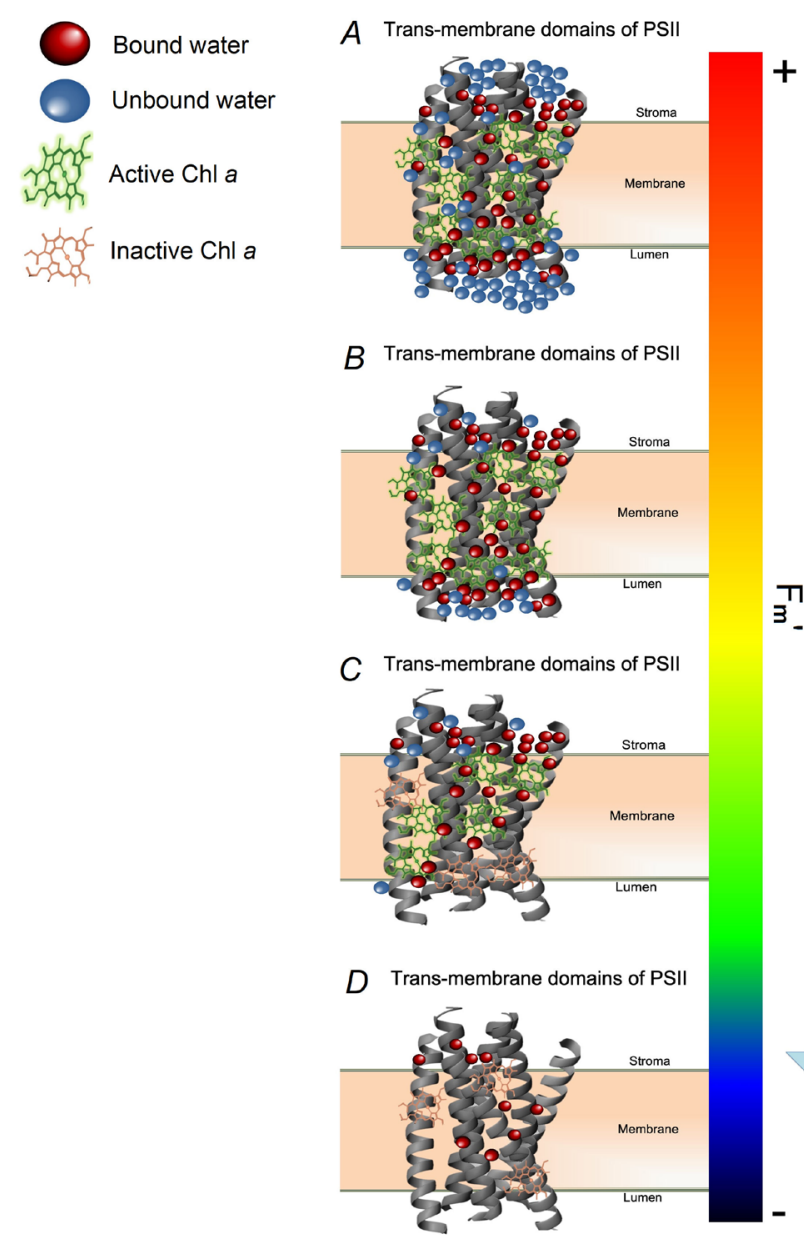

$3_{-}^{T}$

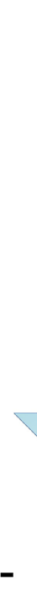

overall ChlF signal (Fig. 6B). The exiting waters will induce some $\mathrm{Chl} a$ molecules to lose their orientation in the structure with consequent variation in the energy partitioning and ChlF. This change will be recoverable and reversible because the unbound waters are highly dynamic across the structure and there is a high probability for them to be replaced when the water potential is restored (e.g., at rewatering). Extremely low water potential in the PSII microenvironment, as during prolonged or severe drought events, will start extracting bound waters as well, leading to unrecoverable levels of ChlF and possible changes in PSII overall stability (Fig. 6C,D). Bound waters are tightly interconnected in the PSII system and less likely to be replaced. This model of interaction corroborates the latest finding for the stability of WSCPs in the Brassicaceae (Palm et al. 2019). When a Chl $a$ is displaced it loses its orientation for the binding site and it has to be considered fully hydrophobic because it will unlikely restabilize to waters. At the same time, newly water-bridged bonds to other position in the protein will not have the strength of the original direct hydrogen bond with consequent alteration for the entire $\mathrm{Chl}$ function.

We emphasize that our conceptual model takes into account one isolated PSII complex (Fig. 6) while leaflevel ChlF is the result of a very large and heterogeneous population of PSII and PSI (Papageorgiou and Govindjee
Fig. 6. Theoretical model of interaction for Chl $a$ and molecular waters impacting ChlF signal. In healthy (fully hydrated) conditions bound (red spheres) and unbound (blue spheres) molecular waters are interconnected to transmembrane proteic domains (gray ribbons) and interact with Chl $a$ molecules (green porphyrin rings) stabilizing the complex $(A)$. At decreasing hydration level (blue arrow, extreme right), first unbound water start leaving the complex causing recoverable changes in the $\mathrm{Chl} a$-water relation $(B)$. With further water depletion, bound waters leave the structure too $(C)$ until $\mathrm{Chl} a$ becomes unstable (brown porphyrin rings) with possible irreparable consequences for the complex (D). The false color scale on the right represents the changes in Chl $a$-water interactions reflected in $\mathrm{ChlF}$, here represented as $\mathrm{F}_{\mathrm{m}}{ }^{\prime}$ as the maximal fluorescence yield of the light-acclimated state.

2004, Kalaji et al. 2017). Future work should address the stoichiometry of the photosystems and the scaling challenges to produce one-to-one inference of $\mathrm{ChlF}$ in this process-model for molecular waters moving in and around PSII. For the same reason, such a mechanistic model has the potential to explain and predict species-specific leaf characteristics (Table 1) that we have here confirmed to largely impact LWC and RChlF. Thus, organ and whole plant process models will be able to incorporate a molecular PSII's hydration mechanism that can then be tied directly to plant hydraulic mechanisms that explain leaf water potential (Sperry and Love 2015). Lastly, this biophysical explanation for the changes in ChlF from PSII under water stress does not disregard the simultaneous activity of NPQ photoprotective processes and chloroplasts' movement to cope with the stress-induced ROS burst (Lichtenthaler and Miehé 1997, Müller et al. 2001, Ruban 2016).

Conclusions: We presented and tested a pipeline to easily gather information on PSII efficiency from high throughput ChlF images that is appropriate to begin testing a molecular mechanism of hydration impacts on PSII. The computed RChlF values from images served to dissect drought progression from mild to moderate to mortality, across diverse species, and they significantly correlated with LWC and RWC throughout the experimental conditions. 
These results opened an exciting prospect of an active role during the stress response for the water molecules present in the PSII complex. In our suggested model, the love-hate relationship between Chl $a$ and water, due to the amphipathic nature of pigment, is critical to the mechanistic interpretation of ChlF dynamics and for its applications at different scales and across species. If validated with future cross-disciplinary experiments, coupling computational molecular dynamics to leaf-level physiology, our model will likely have a large impact on imaging products (and related analyses) captured on cut leaves since the initial leaf water status (bound and unbound waters) can be already compromised in fresh-cut materials, especially in leaves with high succulence. In the future, we see these mechanistic insights on the ChlF signal and its relation to molecular waters to have broad application to all types of ChlF instruments and products, from the most classic PAM fluorometers to hand-held devices, closed/open cameras, and high-throughput phenotyping platforms.

\section{References}

Agostiano A., Cosma P., Trotta M. et al.: Chlorophyll a behavior in aqueous solvents: Formation of nanoscale self-assembled complexes. - J. Phys. Chem. B 106: 12820-12829, 2002.

Anderson J.T., Willis J.H., Mitchell-Olds T.: Evolutionary genetics of plant adaptation. - Trends Genet. 27: 258-266, 2011.

Ashraf M., Mehmood S.: Response of four Brassica species to drought stress. - Environ. Exp. Bot. 30: 93-100, 1990.

Baker N.R.: Chlorophyll fluorescence: A probe of photosynthesis in vivo. - Annu. Rev. Plant Biol. 59: 89-113, 2008.

Baker N.R., Rosenqvist E.: Applications of chlorophyll fluorescence can improve crop production strategies: an examination of future possibilities. - J. Exp. Bot. 55: 1607$1621,2004$.

Barber J., Morris E., Büchel C.: Revealing the structure of the photosystem II chlorophyll binding proteins, CP43 and CP47. - BBA-Bioenergetics 1459: 239-247, 2000.

Battersby A.R.: Tetrapyrroles: the pigments of life. - Nat. Prod. Rep. 17: 507-526, 2000.

Begović L., Galić V., Abičić I. et al.: Implications of intraseasonal climate variations on chlorophyll $a$ fluorescence and biomass in winter barley breeding program. - Photosynthetica 58: 995-1008, 2020.

Beverly D.P., Guadagno C.R., Ewers B.E.: Biophysically informed imaging acquisition of plant water status. - Front. For. Glob. Change 3: 589493, 2020.

Brooks M.D., Niyogi K.K.: Use of a pulse-amplitude modulated (PAM) chlorophyll fluorometer to study the efficiency of photosynthesis in Arabidopsis plants. - In: Jarvis R. (ed.): Chloroplast Research in Arabidopsis. Methods in Molecular Biology. Vol. 775. Pp. 299-310. Humana Press, Totowa 2011.

Butler W.L.: Energy distribution in the photochemical apparatus of photosynthesis. - Ann. Rev. Plant Physio. 29: 345-378, 1978.

Campbell G.S., Smith D.M., Teare B.L.: Application of a dew point method to obtain the soil water characteristic. - In: Schanz T. (ed.): Experimental Unsaturated Soil Mechanics. Springer Proceedings in Physics. Vol. 112. Pp. 71-77. Springer, Berlin-Heidelberg 2007.

Campbell R.J., Mobley K.N., Marini R.P., Pfeiffer D.G.: Growing conditions alter the relationship between SPAD501 values and apple leaf chlorophyll. - HortScience 25: 330-331, 1990.
Chitwood D.H., Sinha N.R.: Evolutionary and environmental forces sculpting leaf development. - Curr. Biol. 26: R297-R306, 2016.

Conkle M.T., Critchfield W.B.: Genetic variation and hybridization of ponderosa pine. - In: Baumgartner D.M., Lotan J.E. (ed.): Ponderosa Pine: The Species and its Management. Pp. $27-$ 44. Washington State University Cooperative Extension, Washington 1988.

Demmig-Adams B., Koh S.-C., Cohu C.M. et al:: Non photochemical quenching in contrasting plant species and environments. - In: Demmig-Adams B., Garab G., Adams III W.W., Govindjee (ed.): Non-Photochemical Quenching and Energy Dissipation in Plants, Algae and Cyanobacteria. Advances in Photosynthesis and Respiration. Vol. 40. Springer, Dordrecht 2014a.

Demmig-Adams B., Stewart J.J., Burch T.A., Adams III W.W.: Insights from placing photosynthetic light harvesting into context. - J. Phys. Chem. Lett. 5: 2880-2889, 2014b.

Fernández-Calleja M., Monteagudo A., Casas A.M. et al.: Rapid on-site phenotyping via field fluorimeter detects differences in photosynthetic performance in a hybrid-parent barley germplasm set. - Sensors-Basel 20: 1486, 2020.

Genty B., Briantais J.-M., Baker N.R.: The relationship between the quantum yield of photosynthetic electron transport and quenching of chlorophyll fluorescence. - BBA-Gen. Subjects 990: 87-92, 1989.

Giles K., Beardsell M.F., Cohen D.: Cellular and ultrastructural changes in mesophyll and bundle sheath cells of maize in response to water stress. - Plant Physiol. 54: 208-212, 1974.

Girr P., Kilper J., Pohland A.-C., Paulsen H.: The pigment binding behavior of water-soluble chlorophyll protein (WSCP). Photoch. Photobio. Sci. 19: 695-712, 2020.

Gorbe E., Calatayud A.: Applications of chlorophyll fluorescence imaging technique in horticultural research: A review. - Sci. Hortic.-Amsterdam 138: 24-35, 2012.

Govindjee G.: Chlorophyll a fluorescence: a bit of basics and history. - In: Papageorgiou G.C., Govindjee G. (ed.): Chlorophyll $a$ Fluorescence. Advances in Photosynthesis and Respiration. Vol. 19. Pp. 1-42. Springer, Dordrecht 2004.

Gu L., Han J., Wood J.D. et al:: Sun-induced Chl fluorescence and its importance for biophysical modeling of photosynthesis based on light reactions. - New Phytol. 223: 1179-1191, 2019.

Guadagno C.R., Ewers B.E.: Chlorophyll $a$ fluorescence analyses to investigate the impacts of genotype, species and stress on photosynthetic efficiency and plant productivity. - In: Samal A., Choudhury S.D. (ed.): Intelligent Image Analysis for Plant Phenotyping. Pp. 26. CRC Press, Boca Raton 2020.

Guadagno C.R., Ewers B.E., Speckman H.N. et al.: Dead or alive? Using membrane failure and chlorophyll $a$ fluorescence to predict plant mortality from drought. - Plant Physiol. 175: 223-234, 2017.

Guadagno C.R., Ewers B.E., Weinig C.: Circadian rhythms and redox state in plants: till stress do us part. - Front. Plant Sci. 9: 247, 2018.

Guidi L., Tattini M., Landi M.: How does the chloroplast protect chlorophyll against excessive light? - In: Jacob-Lopes E., Queiroz Zepka L., Queiroz M.I. (ed.): Chlorophyll. InTech, 2017.

Herritt M.T., Pauli D., Mockler T.C., Thompson A.L.: Chlorophyll fluorescence imaging captures photochemical efficiency of grain sorghum (Sorghum bicolor) in a field setting. - Plant Methods 16: 109, 2020.

Kalaji H.M., Jajoo A., Oukarroum A. et al.: Chlorophyll a fluorescence as a tool to monitor physiological status of plants under abiotic stress conditions. - Acta Physiol. Plant. 38: 102, 2016. 
Kalaji H.M., Schansker G., Brestic M. et al.: Frequently asked questions about chlorophyll fluorescence, the sequel. Photosynth. Res. 132: 13-66, 2017.

Koide R.T., Robichaux R.H., Morse S.R., Smith C.M.: Plant water status, hydraulic resistance and capacitance. - In: Pearcy R.W., Ehleringer J.R., Mooney H.A., Rundel P.W. (ed.): Plant Physiological Ecology. Pp. 161-183. Springer, Dordrecht 1989.

Kuhlgert S., Austic G., Zegarac R. et al:: MultispeQ Beta: A tool for large-scale plant phenotyping connected to the open PhotosynQ network. - Royal Soc. Open Sci. 3: 160592, 2016.

Lichtenthaler H.K.: The stress concept in plants: an introduction. - Ann. N.Y. Acad. Sci. 851: 187-198, 1998.

Lichtenhaler H.K., Buschmann C., Rinderle U., Schmuck G.: Application of chlorophyll fluorescence in ecophysiology. Radiat. Environ. Bioph. 25: 297-308, 1986.

Lichtenthaler H.K., Langsdorf G., Lenk S., Buschmann C.: Chlorophyll fluorescence imaging of photosynthetic activity with the flash-lamp fluorescence imaging system. Photosynthetica 43: 355-369, 2005.

Lichtenthaler H.K., Miehé J.A.: Fluorescence imaging as a diagnostic tool for plant stress. - Trends Plant Sci. 2: 316320, 1997.

Lide D.R. (ed.): CRC Handbook of Chemistry and Physics. $90^{\text {th }}$ Edition. Pp. 2804. CRC Press, Boca Raton 2009.

Lin K., Zhang N., Severing E.I. et al.: Beyond genomic variation - comparison and functional annotation of three Brassica rapa genomes: a turnip, a rapid cycling and a Chinese cabbage. - BMC Genomics 15: 250, 2014.

Linke K., Ho F.M.: Water in Photosystem II: Structural, functional and mechanistic considerations. - BBA-Bioenergetics 1837: 14-32, 2014.

Magney T.S., Barnes M.L., Yang X.: On the covariation of chlorophyll fluorescence and photosynthesis across scales. Geophys. Res. Lett. 47: e2020GL091098, 2020.

Marenco R.A., Antezana-Vera S.A., Nascimento H.C.S.: Relationship between specific leaf area, leaf thickness, leaf water content and SPAD-502 readings in six Amazonian tree species. - Photosynthetica 47: 184-190, 2009.

Matsubara S., Chow W.S.: Populations of photoinactivated photosystem II reaction centers characterized by chlorophyll $a$ fluorescence lifetime in vivo. - P. Natl. Acad. Sci. USA 101: 18234-18239, 2004.

Maxwell K., Johnson G.N.: Chlorophyll fluorescence a practical guide. - J. Exp. Bot. 345: 659-668, 2000.

Mirkovic T., Ostroumov E.E., Anna J.M. et al.: Light absorption and energy transfer in the antenna complexes of photosynthetic organisms. - Chem. Rev. 117: 249-293, 2017.

Müller P., Li X.-P., Niyogi K.K.: Non-photochemical quenching. A response to excess light energy. - Plant Physiol. 125: 15581566, 2001.

Murchie E.H., Lawson T.: Chlorophyll fluorescence analysis: a guide to good practice and understanding some new applications. - J. Exp. Bot. 64: 3983-3998, 2013.

Murphy R.J., Whelan B., Chlingaryan A., Sukkarieh S.: Quantifying leaf-scale variations in water absorption in lettuce from hyperspectral imagery: a laboratory study with implications for measuring leaf water content in the context of precision agriculture. - Precis. Agric. 20: 767-787, 2019.

Palm D.M., Agostini A., Pohland A.-C.: Stability of water-soluble chlorophyll protein (WSCP) depends on phytyl conformation.ACS Omega 4: 7971-7979, 2019.

Papageorgiou G.C., Govindjee G. (ed.): Chlorophyll a Fluorescence: A Signature of Photosynthesis. Advances in Photosynthesis and Respiration. Pp. 818. Springer, Dordrecht 2004.
Porcar-Castell A., Tyystjärvi E., Atherton J. et al.: Linking chlorophyll $a$ fluorescence to photosynthesis for remote sensing applications: mechanisms and challenges. - J. Exp. Bot. 65: 4065-4095, 2014.

Radermacher A.L., du Toit S.F., Farrant J.M.: Desiccation-driven senescence in the resurrection plant Xerophyta schlechteri (Baker) N.L. Menezes: Comparison of anatomical, ultrastructural, and metabolic responses between senescent and non-senescent tissues. - Front. Plant Sci. 10: 1396, 2019.

Raven P.H., Evert R.F., Eichhorn S.E.: Photosynthesis, Light, and Life. - In: Raven P.H., Evert R.F., Eichhorn S.E. (ed.): Biology of Plants. $7^{\text {th }}$ Edition. Pp. 119-127. W.H. Freeman \& Company, New York 2004.

Richardson A.D., Duigan S.P., Berlyn G.P.: An evaluation of noninvasive methods to estimate foliar chlorophyll content. New Phytol. 153: 185-194, 2002.

Ruban A.V.: Nonphotochemical chlorophyll fluorescence quenching: Mechanism and effectiveness in protecting plants from photodamage. - Plant Physiol. 170: 1903-1916, 2016.

Saito K., Mitsuhashi K., Ishikita H.: Dependence of the chlorophyll wavelength on the orientation of a charged group: Why does the accessory chlorophyll have a low site energy in photosystem II? - J. Photoch. Photobio. A 402: 112799, 2020.

Schansker G., Tóth S.Z., Strasser R.J.: Methylviologen and dibromothymoquinone treatments of pea leaves reveal the role of photosystem I in the Chl $a$ fluorescence rise OJIP. BBA-Bioenergetics 1706: 250-261, 2005.

Scoffoni C., Vuong C., Diep S. et al.: Leaf shrinkage with dehydration: coordination with hydraulic vulnerability and drought tolerance. - Plant Physiol. 164: 1772-1788, 2014.

Shen J.R.: The structure of photosystem II and the mechanism of water oxidation in photosynthesis. - Annu. Rev. Plant Biol. 66: 23-48, 2015.

Shipman L.L., Cotton T.M., Norris J.R., Katz J.J.: New proposal for structure of special-pair chlorophyll. - P. Natl. Acad. Sci. USA 73: 1791-1794, 1976.

Sperry J.S., Love D.M.: What plant hydraulics can tell us about plant responses to climate-change droughts. - New Phytol. 207: 14-17, 2015.

Sun H., Feng M., Xiao L. et al:: Assessment of plant water status in winter wheat (Triticum aestivum L.) based on canopy spectral indices. - PLoS ONE 14: e0216890, 2019.

Tietz S., Hall C.C., Cruz J.A., Kramer D.M.: $\mathrm{NPQ}_{(\mathrm{T})}$ : A chlorophyll fluorescence parameter for rapid estimation and imaging of non-photochemical quenching of excitons in photosystem-II-associated antenna complexes. - Plant Cell Environ. 40: 1243-1255, 2017.

Uddling J., Gelang-Alfredsson J., Piikki K., Pleijel H.: Evaluating the relationship between leaf chlorophyll concentration and SPAD502 chlorophyll meter readings. - Photosynth. Res. 91: 37-46, 2007.

Urban L., Aarrouf J., Bidel L.P.R.: Assessing the effects of water deficit on photosynthesis using parameters derived from measurements of leaf gas exchange and of chlorophyll $a$ fluorescence. - Front Plant Sci. 8: 2068, 2017.

van Bezouwen L.S., Caffarri S., Kale R.S. et al.: Subunit and chlorophyll organization of the plant photosystem II supercomplex. - Nat. Plants 3: 17080, 2017.

Vasil'ev S., Shen J.-R., Kamiya N., Bruce D.: The orientations of core antenna chlorophylls in photosystem II are optimized to maximize the quantum yield of photosynthesis. - FEBS Lett. 561: 111-116, 2004.

Vogt L., Vinyard D.J., Khan S., Brudvig G.W.: Oxygen evolving complex of Photosystem II: an analysis of second-shell residues and hydrogen bonding networks. - Curr. Opin. Chem. Biol. 25: 152-158, 2015. 


\section{DEPENDENCY OF THE ChlF SIGNAL UPON LEAF HYDRATION}

Vredenberg W.J.: A three state model for energy trapping and chlorophyll fluorescence in Photosystem II incorporating radical pair recombination. - Biophys. J. 79: 26-38, 2000.

Weatherley P.E.: Studies in water relations of cotton plants. I. The field measurement of water deficit in leaves. - New Phytol. 49: 81-87, 1950.

Woo N.S., Badger M.R., Pogson B.J.: A rapid, non-invasive procedure for quantitative assessment of drought survival using chlorophyll fluorescence. - Plant Methods 4: 27, 2008. Wright I., Reich P., Westoby M. et al.: The worldwide leaf economics spectrum. - Nature 428: 821-827, 2004.

Yao J., Sun D., Cen H. et al.: Phenotyping of Arabidopsis drought stress response using kinetic chlorophyll fluorescence and multicolor fluorescence imaging. - Front. Plant Sci. 9: 603, 2018.

(C) The authors. This is an open access article distributed under the terms of the Creative Commons BY-NC-ND Licence. 